\title{
Oral lichen planus and malignant transformation: The role of p16, Ki-67, Bub-3 and SOX4 in assessing precancerous potential
}

\author{
EDUARDO AUGUSTO ROSA ${ }^{1 *}$, AURA MARIA HURTADO-PUERTO ${ }^{2 *}$, DENISE PINHEIRO FALCÃO ${ }^{1}$, \\ ALINE PATRICIA BRIETZKE ${ }^{3}$, LUIZ EDUARDO DE ALMEIDA PRADO FRANCESCHI ${ }^{4}$, \\ FLORLORÊNCIO FIGUEIREDO CAVALCANTI NETO ${ }^{1}$, VALDENIZE TIZIANE ${ }^{5}$, FABIANA PIRANI CARNEIRO ${ }^{1}$, \\ EVELYN MIKAELA KOGAWA ${ }^{1}$, HEITOR MORENO ${ }^{6}$ and RIVADÁVIO FERNANDES BATISTA AMORIM ${ }^{1}$ \\ ${ }^{1}$ Post-Graduation Program in Medical Sciences, Department of Pathology, School of Medicine, \\ University of Brasília, Brasília 70910-900, Brazil; ${ }^{2}$ Laboratory for Neuropsychiatry and Neuromodulation, \\ Department of Psychiatry, Massachusetts General Hospital, Boston, MA 02114, USA; ${ }^{3}$ Laboratory of Pain and \\ Neuromodulation at Hospital de Clínicas de Porto Alegre, Federal University of Rio Grande do Sul, \\ Porto Alegre, Rio Grande do Sul 90035-903; ${ }^{4}$ Department of Pathology, School of Medicine, University of Brasília; \\ ${ }^{5}$ Center for Learning and Research, Brasília Children's Hospital, Brasília 70910-900; ${ }^{6}$ Laboratory of Cardiovascular \\ Pharmacology, School of Medical Sciences, University of Campinas, Campinas, São Paulo 13083-970, Brazil
}

Received October 31, 2016; Accepted December 8, 2017

DOI: $10.3892 /$ etm.2018.5971

\begin{abstract}
The association of oral lichen planus (OLP) lesions with malignant transformation risk has remained a controversial topic and is of clinical importance. Therefore, the present study evaluated the expression levels of p16, Ki-67, budding uninhibited by benzimidazoles 3 (Bub-3) and sex-determining region Y-related high mobility group box 4 (SOX4), and their roles as precancerous biomarkers in OLP. A retrospective study was performed, in which tissue blocks of OLP, oral dysplasia (OD), cutaneous lichen planus (CLP) and oral fibrous hyperplasia $(\mathrm{OFH})$ were used $(\mathrm{n}=120)$. A positivity index $(\mathrm{PI})$ for p16, BUB3, Ki-67 and SOX4 expression was calculated in each group. The PI for p16 was $20.65 \%$ for OLP, $7.85 \%$ for OD, $86.59 \%$ for CLP and $11.8 \%$ for OFH, and the difference between these groups was statistically significant $(\mathrm{P}<0.001)$. PIs of Ki-67 were indicated as $11.6 \%$ for OLP, $14.4 \%$ for OD, 8.24\% for CLP and 5.5\% for OFH, and a statistically significant difference was observed between the groups $(\mathrm{P}<0.001)$. Notably, the expression levels of BUB3 were not statistically different among groups. The highest expression levels of
\end{abstract}

Correspondence to: Dr Rivadávio Fernandes Batista Amorim, Post-Graduation Program in Medical Sciences, Department of Pathology, School of Medicine, University of Brasília, Campus Darcy Ribeiro, Asa Norte, Brasília 70910-900, Brazil

E-mail: rivadavioamorim@hotmail.com

${ }^{*}$ Contributed equally

Key words: lichen planus, oral lichen planus, precancerous conditions, p16 (INK4A), Ki-67 antigen, Bub-3, SOX4, biomarkers
SOX4 were identified in CLP $(\mathrm{P}<0.001$ vs. OLP/CLP; $\mathrm{P}=0,001$ vs. CLP/OD). The determined expression levels of p16 and Ki-67 suggest that specific OLP lesions may have an intermediate malignant potential and should be carefully followed up. The intense SOX4 staining in CLP indicated a different proliferation pattern of epithelium compared with oral mucosa cells. These findings suggest that SOX4 expression may also be associated with the different clinical courses of OLP and CLP.

\section{Introduction}

Oral cancer is considered one of the most common cancer types around the world (1). The estimated incidence of oral cancer and associated fatalities per year worldwide are 300,000 and 145,000 , respectively (1). Typically, oral squamous cell carcinoma (OSCC) is preceded by epithelial precancerous lesions, which are defined as morphologically altered tissue in which cancer is more likely to occur compared with its normal mucosae (2). The presence of epithelial oral dysplasia (OD) in precancerous lesions is an indicator of malignancy transformation (3). Early diagnosis of such lesions may therefore aid in preventing the emergence of OSCC and ameliorating the burden of the disease $(2,3)$.

Lichen planus is one of the most common inflammatory mucocutaneous diseases with an incidence of $0.5-2.0 \%$ in the adult population and a female to male ratio of 3:1 (4). Lichen planus affects the skin, mucosa (including the oral mucosa) or a combination of both (4). In 1978, the World Health Organization (WHO) indicated that oral lichen planus (OLP) is a precancerous condition (5). In this regard, the association between OLP and oral cancer has been extensively studied. However, whether OLP lesions have an increased risk of malignant transformation and the extent of this remains a controversial issue (6-8). 
The identification of reliable biomarkers for detecting malignant transformation poses a unique role for the development of standardized screening and improved follow-up in patients with oral precancerous lesions. There is a good perspective for the feasible coupling of these techniques with other strategies, such as multimodal cell analysis for brush biopsies in the early detection of potentially premalignant lesions (9). The antibody for the nuclear antigen $\mathrm{Ki}-67$ is a well-established marker of cell proliferation and has been widely studied in OSCC and precancerous oral lesions $(10,11)$. Although Ki-67 has been evaluated as a risk factor in the development of oral cancer from precancerous lesions, only a limited number of studies have explored the expression of Ki-67 in OLP in comparison with other epithelial lesions $(10,11)$. In addition, previous results have suggested the diagnostic and predictive value of p16 in different types of cancer, particularly in that of the cervix (12). Notably, P16 is a cell cycle progression inhibitor that is involved in the inhibition of cyclin-dependent kinase (CDK)4 and CDK6 (12). Despite this important role in the cell cycle, its value as a predictor of malignancy progression remains controversial and is dependent on the anatomical site; for example, in cervical or breast cancer (12).

Various novel potential markers have been studied for their association with malignancy in preclinical and clinical trials. For example, budding uninhibited by benzimidazoles 3 (Bub-3) is known as a mitotic checkpoint gene that inhibits mitosis (13). The expression of this gene has been associated with low-grade, as opposed to high-grade, luminal breast cancer (14). Furthermore, mutations in BUB3 have been associated with colorectal cancer (15). Additionally, the overexpression of the transcription factor sex-determining region Y-related high mobility group box 4 (SOX4), which is also considered a novel proliferation marker, is hypothesized to be an unfavorable prognostic factor in patients with breast cancer (16). SOX4 is broadly expressed in small-cell lung carcinoma and esophageal squamous cell carcinoma, and has therefore been considered as a potential target for lung cancer vaccines $(16,17)$.

The aim of the present study was to evaluate the expression levels of p16, Ki-67, Bub-3 and SOX4 in order to assess their role as potential markers of malignant transformation in OLP. To the best of our knowledge, no studies thus far have determined the expression of Bub-3 and SOX4 in OLP. The analysis of these expression levels may elucidate the mechanism and provide novel evidence concerning malignant transformation in OLP lesions.

\section{Materials and methods}

Study design. In the present retrospective study design, biopsy tissue samples of lesions of OLP, OD, cutaneous lichen planus (CLP) and oral fibrous hyperplasia (OFH) were obtained from 120 patients (44 males and 76 females; mean age, 49 years), prior to being fixed in $10 \%$ formalin ( $24 \mathrm{~h}$ at room temperature) and paraffin-embedded for a maximum of 5 years. The patient selection was made by convenience, according to the given criteria for each study group, as described below. The data from each subject, including age, sex and lesion location were collected using medical record files of the Brasília University Hospital, Brasília, Brazil. The following exclusion criteria were applied: Lack of data regarding clinical diagnosis, divergence between clinical and histological aspects and poor amount of material for immunohistochemical analysis. The present study was reviewed and approved by the Institutional Review Board (IRB) from the School of Medicine, University of Brasília (Brasília, Brazil; approval number, CEPFM 042/2010) and conducted in accordance with the principles of the Declaration of Helsinki. Informed consent was obtained in the majority of cases; however, some patients were not available following several contact attempts or had experienced mortality. Therefore, the IRB approved not having the formal consent in these cases. In order to improve the evaluation of the present study, the inclusion of specific groups was incorporated. CLP was selected as the negative control as it is the skin counterpart of the disease that is not associated with malignant transformation $(6,8)$. The most well-known oral lesions that have a tendency towards cancer progression are OD lesions (5) and therefore this was used as the positive control. OFH was also considered in the present study as negative control, despite being reactive lesions with increased proliferation, the risk of malignancy progression is negligible (5).

Samples. The diagnosis of OLP and CLP was based on clinical and histological features. All OLP cases exhibited bilateral involvement and were divided into three subtypes: Reticular, when white plaques and/or reticular lesions were reported; erythematous, when erythematous areas (reddish in color) were described; and erosive (eroOLP), when ulcerations were added to the previous features. The histologic criteria for OLP diagnosis was determined as previously described (18). As exclusion criteria, no signs of epithelial dysplasia could be present in cases classified as OLP. The same histological parameters were used for the CLP group. Additionally, histological criteria used for selection of OD cases were as previously defined by WHO (5). Lesions were categorized as $\mathrm{OFH}$ when there was a detection of proliferative fibrous connective tissue with dense collagen arrangement that was lined by squamous epithelium.

Immunohistochemistry. Immunohistochemistry was performed on all tissue samples, using the streptavidin-biotin method. Information associated with the primary antibodies used, including the manufacturer, clone, dilution, incubation time, temperature and antigen retrieval buffer is summarized in Table I. All primary antibodies were diluted in $1 \%$ buffered bovine serum albumin (Sigma-Aldrich; Merck KGaA, Darmstadt Germany). Sections (4- $\mu$ m thick) from paraffin-embedded blocks were deparaffinized and rehydrated, then fixed in a mixture of methanol and acetone for $15 \mathrm{~min}$ at room temperature. Specimens were then immersed in antigen retrieval buffer and incubated for $20 \mathrm{~min}$ at $98^{\circ} \mathrm{C}$. Endogenous peroxidase activity was blocked using $0.3 \%$ hydrogen peroxide, for $20 \mathrm{~min}$ at room temperature. The primary antibodies were detected using the Polyvalent HRP Plus kit (Spring Bioscience Corporation, Pleasanton, CA, USA) and 3,3'-diaminobenzidine tetrahydrochloride chromogen with 2 drops at room temperature for 10 min (Dako; Agilent Technologies, Inc., Santa Clara, CA, USA). Incubation 
Table I. Primary features of the immunohistochemical antibodies p16, Ki-67, BUB3 and SOX4.

\begin{tabular}{|c|c|c|c|c|c|c|}
\hline Antibody & Clone & Manufacturer & Dilution & $\begin{array}{l}\text { Incubation } \\
\left(\mathrm{h} /{ }^{\circ} \mathrm{C}\right)\end{array}$ & $\begin{array}{l}\text { Antigen } \\
\text { retrieval }\end{array}$ & $\begin{array}{l}\text { Positive } \\
\text { controls }\end{array}$ \\
\hline p16 & G175-405 & $\begin{array}{l}\text { BD Pharmingen; BD Biosciences, } \\
\text { San Jose, CA, USA }\end{array}$ & $1: 200$ & $18 / 4$ & Citrate $\mathrm{pH} 6.0$ & $\begin{array}{l}\text { Cervical } \\
\text { carcinoma }\end{array}$ \\
\hline Ki-67 & SP6 & $\begin{array}{l}\text { Biocare Medical, Concord, } \\
\text { CA, USA }\end{array}$ & $1: 50$ & $18 / 4$ & Citrate $\mathrm{pH} 6.0$ & Palatine tonsil \\
\hline BUB3 & EPR5319 (2) & Abcam, Cambridge, MA, USA & $1: 500$ & $18 / 4$ & Citrate $\mathrm{pH} 6.0$ & Breast gland \\
\hline SOX4 & Polyclonal & Abcam, Cambridge & $1: 800$ & $18 / 4$ & Citrate $\mathrm{pH} 6.0$ & Breast gland \\
\hline
\end{tabular}

SOX4, sex determining region Y-box 4; BUB3, budding uninhibited by benzimidazoles 3 .

with secondary antibodies using a Dako Cytomation LSAB System-HRP Kit (Dako Agilent Technologies, Inc., Santa Clara, CA, USA) was conducted in a humid chamber at $37^{\circ} \mathrm{C}$ for $1 \mathrm{~h}$. A light microscope was used to observe the slides at a magnification of $x 400$.

Evaluation of Ki-67, pl6, Bub-3 and SOX4 expression levels. Histological images were acquired using a ScanScope CS System histological scanner (Aperio ePathology Solutions; Leica Microsystems GmbH, Wetzlar, Germany) at a magnification of x400 (resolution: $0.25 \mu / \mathrm{pixel}$ ), using ImageScope (Leica Microsystems $\mathrm{GmbH}$ ). Only histological areas that indicated a typical appearance of the studied lesions were assessed. For instance, a field of OFH in which epithelium hyperplasia was not detected indicated that this field was not eligible for further analysis. Following this initial histological screening, at least six fields were randomly and systematically selected for each case. Positive and negative epithelial cells were counted using ImageJ version $1.51 \mathrm{~g}$; Software (National Institutes of Health, Bethesda, MD, USA). Quantitative assessment was performed by counting 500 cells at the basal and suprabasal epithelial cell layers in each case and for each biomarker studied. The percentage of positive cells (n/500) was obtained and recorded as the positivity index (PI) for each marker. Epithelial cells indicating nuclear brown staining were considered positive for BUB3 and Ki-67. Nuclear or cytoplasmic staining was considered positive for p16 and SOX4 antibodies.

Statistical analysis. Data analyses were performed using SPSS version 20.0 for Windows (IBM Corp., Armonk, NY, USA). All tests were two-tailed and $\mathrm{P}<0.05$ was considered to indicate a statistically significant difference. The Kolmogorov-Smirnov test was used to determine the frequency distribution of the variables. In cases of normal distribution, a one-way analysis of variance with pairwise comparisons was performed. The Tukey's post-hoc test was performed when statistical differences were identified, if necessary. For non-normal distribution, Kruskal-Wallis test was applied to detect any difference among groups associated with continuous variables and the Dunn post-hoc test was applied when statistical significance was indicated. The $\chi^{2}$ test was used to assess differences associated with categorical variables, such as sex, among groups. Spearman's correlation tests were used to evaluate the expression of the four antibodies studied.

\section{Results}

General data. A total of 120 paraffin block samples were evaluated that were equally distributed into the OLP $(n=30)$, OD $(n=30)$, OFH $(n=30)$ and CLP $(n=30)$ groups. Results are indicated in Table II. No significant differences between the demographic characteristics were revealed. The mean age of subjects and the female-to-male ratio demonstrated no significant differences among groups $(\mathrm{P}=0.71$ and $\mathrm{P}=0.83$, respectively).

Immunohistochemical data are summarized in Figs. 1 and 2. In the following text, PI is represented in percentage (\%) and the 25 and 75 th percentiles follow in parenthesis. Fig. 3 indicates case no. 22 and presents the expression of P16, BUB3, SOX4 and Ki67 in OLP. In this particular case, Bub-3 demonstrated the highest level of immunostaining. Fig. 4 indicates case no. 12 and presents the expression of p16, Bub-3, SOX4 and $\mathrm{Ki}-67$ in CLP. In this case, all antibodies demonstrated strong immunostaining with the exception of Ki67, which stained weakly. Fig. 5 presents case no. 29, demonstrating the expression of p16, BUB3, SOX4 and Ki-67 in OD. All antibodies exhibited strong immunostaining with the exception of SOX4, which was only moderately stained. Fig. 6 presents case no. 23 and demonstrates the expression of P16, BUB3, SOX4 and Ki67 in OFH. Bub-3 and SOX4 exhibited strong immunostaining.

P16 data. The PI for p16 was $20.65 \%$ (14.02-23.95) for OLP, $7.85 \%$ (5.22-18.12) for OD, 86.59\% (49.80-91.80) for CLP and $11.8 \%(6.82-18.50)$ for $\mathrm{OFH}$, and the difference between these groups was statistically significant $(\mathrm{P}<0.001)$. Notably, the Dunn post hoc test indicated a significant difference between OLP and OD $(\mathrm{P}<0.002)$. Although the distribution was not statistically significant, the expression of p16 by OLP macroscopic type was unevenly distributed (Fig. 2). The widest distribution was observed in the eroOLP type $(\mathrm{P}=0.403)$.

$\mathrm{Ki}-67$ data. PIs of Ki-67 were indicated as $11.6 \%(5.60-15.15)$ for OLP, $14.4 \%(11.25-22.8)$ for OD, $8.24 \%$ (5.40-13.45) for CLP and $5.5 \%(3.00-7.45)$ for OFH, and a statistically 
Table II. Demographic and clinical characteristics of each study group.

\begin{tabular}{|c|c|c|c|c|}
\hline \multirow[b]{2}{*}{ Characteristics } & \multicolumn{3}{|c|}{ Oral lesions } & \multirow{2}{*}{$\frac{\text { Skin lesions }}{\begin{array}{c}\text { Cutaneous lichen planus } \\
(\mathrm{n}=30)\end{array}}$} \\
\hline & $\begin{array}{l}\text { Oral lichen planus } \\
\qquad(\mathrm{n}=30)\end{array}$ & $\begin{array}{l}\text { Oral dysplasia } \\
\qquad(\mathrm{n}=30)\end{array}$ & $\begin{array}{l}\text { Oral fibrous hyperplasia } \\
\qquad(\mathrm{n}=30)\end{array}$ & \\
\hline \multicolumn{5}{|l|}{ Age, years } \\
\hline Mean & 51.8 & 49.4 & 49 & $47.2^{\mathrm{a}}$ \\
\hline $95 \%$ confidence interval & $46.2-57.1$ & $43.2-55.6$ & $43.6-54.4$ & $41.9-52.4$ \\
\hline \multicolumn{5}{|l|}{ Sex, n (\%) } \\
\hline Male & $9(30)$ & $12(40)$ & $11(36.7)$ & $12(40)^{\mathrm{b}}$ \\
\hline Female & $21(70)$ & $18(60)$ & $19(63.7)$ & $18(60)$ \\
\hline \multicolumn{5}{|l|}{ Biopsy site, n (\%) } \\
\hline Buccal mucosa & $15(50)$ & $3(10)$ & $5(16.7)$ & - \\
\hline Lips & $2(6.7)$ & $5(16.7)$ & $2(6.7)$ & - \\
\hline Gums & $3(10)$ & $2(6.7)$ & $2(6.7)$ & - \\
\hline Tongue & $3(10)$ & $3(10)$ & $1(3.3)$ & - \\
\hline Palate & $1(3.3)$ & $3(10)$ & $2(6.7)$ & - \\
\hline Buccal fornix & $2(6.7)$ & - & $2(6.7)$ & - \\
\hline Retromolar triangle & - & $1(3.3)$ & $1(3.3)$ & - \\
\hline Alveolar ridge & - & - & $1(3.3)$ & - \\
\hline Floor of the mouth & - & $1(3.3)$ & - & - \\
\hline Upper limbs & - & - & - & $7(23.3)$ \\
\hline Trunk & - & - & - & $5(16.3)$ \\
\hline Lower limbs & - & - & - & $4(13.3)$ \\
\hline Neck & - & - & - & $3(10)$ \\
\hline Penis & - & - & - & $1(3.3)$ \\
\hline Face & - & - & - & $1(3.3)$ \\
\hline Head & - & - & - & $1(3.3)$ \\
\hline Not available & $4(13.3)$ & $12(40)$ & $14(46.7)$ & $8(26.7)$ \\
\hline Total & $30(100)$ & $30(100)$ & $30(100)$ & $30(100)$ \\
\hline
\end{tabular}

${ }^{\mathrm{a} A g e}$ among oral lichen planus, oral dysplasia, oral fibrous hyperplasia and cutaneous lichen planus; $\mathrm{P}=0.71$ (analysis of variance); ${ }^{\mathrm{b}} \mathrm{Sex}$ among oral lichen planus, oral dysplasia, oral fibrous hyperplasia and cutaneous lichen planus $\mathrm{P}=0.83$ ( $\chi^{2}$ test).

significant difference was observed between groups $(\mathrm{P}<0.001)$. Dunn post hoc tests indicated that a significant difference existed between OLP and OFH $(\mathrm{P}<0.005)$, and between OD and $\mathrm{OFH}(\mathrm{P}=0.012)$.

Bub-3 data. Assessment of BUB3 expression resulted in PIs of $80.0 \%$ (60.15-88.30) for OLP, $71.4 \%$ (38.10-79.75) for OD, $83.2 \%$ (60.95-89.40) for CLP and 77\% (53.10-91.90) for $\mathrm{OFH}$, and a statistically significant difference between these groups was indicated $(\mathrm{P}=0.04)$. Pairwise comparisons demonstrated a significant difference between only CLP and OD $(\mathrm{P}=0.04)$.

SOX4 data. PIs for SOX4 were 66.1\% (11.20-86.20) for OLP, $72.4 \%(61.05-82.55)$ for OD, 86.8\% (78.65-92.4) for CLP and $82.3 \%(71.95-88.65)$ for $\mathrm{OFH}$, and a statistically significant difference between all groups was demonstrated $(\mathrm{P}<0.001)$. Pairwise comparisons revealed a significant difference between OLP and CLP $(\mathrm{P}<0.001)$ and between CLP and OD $(\mathrm{P}=0.001)$.

\section{Discussion}

To the best of our knowledge, the present study is the first to evaluate the expression of p16, Ki-67, Bub-3 and SOX4 as markers of malignant transformation in OLP. To explore the roles of these factors in OLP, OFH was considered as a negative control for oral malignancy (proliferative benign lesion), OD was regarded as a standard of pre-malignant lesion and CLP was used as the skin counterpart of OLP with no tendency to malignancy thus also a negative control. The present results provide indications of the possible mechanisms associated with the nature of OLP and the association of p16 and Ki-67 expression with OLP.

In the present study, the criteria for selecting the groups was defined by separating lesions with a low risk of malignant transformation of dysplastic epithelium lesions and those with malignant potential and these were compared with OLP. Although OD is not considered a lesion, it is the most well-known factor that has the potential for malignant transformation (5). 

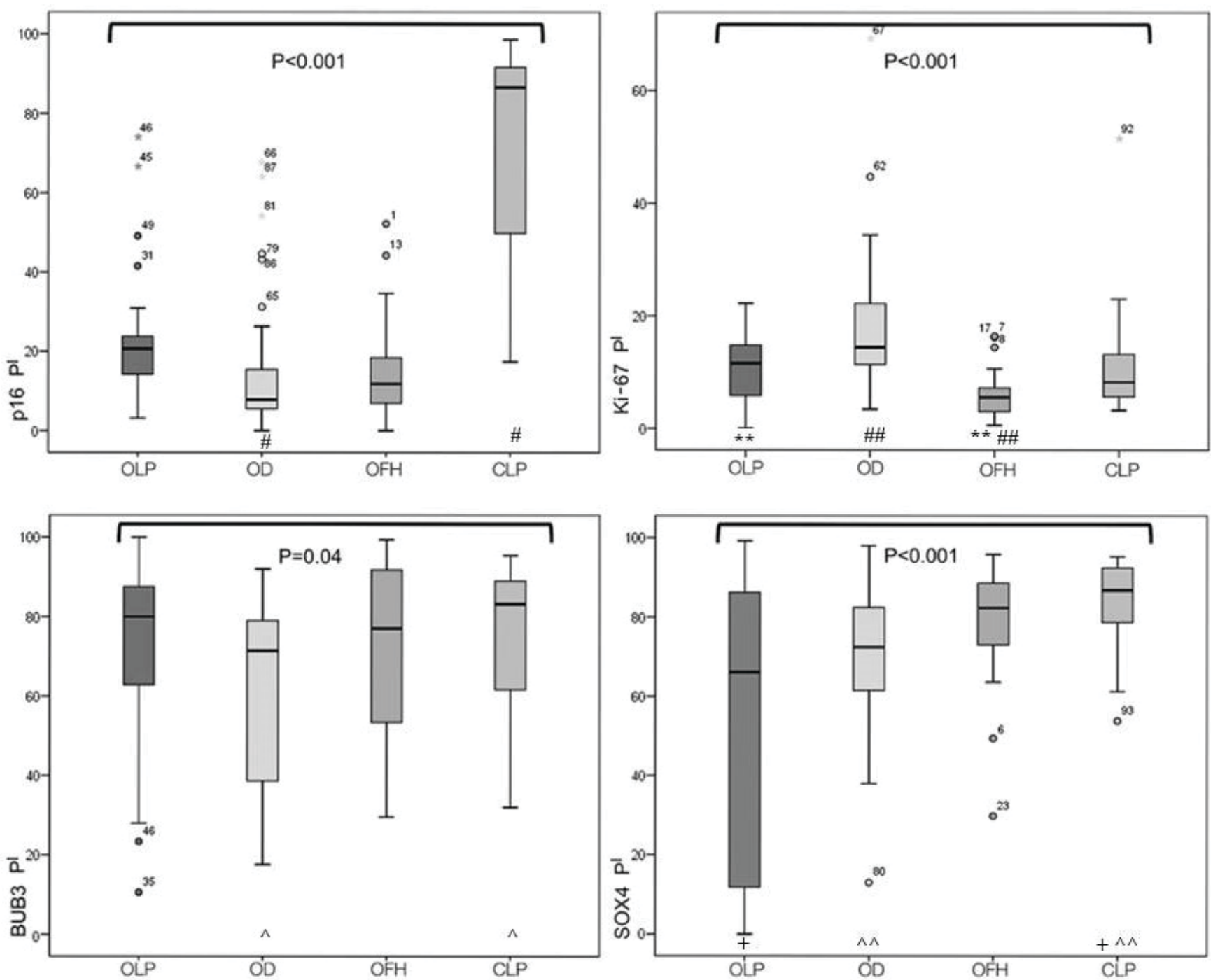

Figure 1. Expression of p16, Ki-67, BUB3 and SOX4 antibodies in OLP, OD, OFH and CLP. Differences among all antibodies were determined using the Kruskal-Wallis test ( $\mathrm{P}$-values written in full). The groups with indicators represent $\mathrm{P}$-values that were determined following the Dunn's post hoc test: ${ }^{\#} \mathrm{P}<0.002$; ${ }^{* *} \mathrm{P}<0.005 ;{ }^{\# \#} \mathrm{P}=0.012 ;{ }^{\wedge} \mathrm{P}=0.04 ;{ }^{+} \mathrm{P}<0.001 ;{ }^{\wedge} \mathrm{P}=0.001$. SOX4, sex determining region Y-box 4; BUB3, budding uninhibited by benzimidazoles 3 ; OLP, oral lichen planus; OD, oral dysplasia; OFH, oral fibrous hyperplasia; CLP, cutaneous lichen planus; PI, positivity index (percentage of positive cells n/500).

p16, a protein identified as a part of the retinoblastoma $(\mathrm{Rb})$ pathway, has a key role in cell cycle regulation and binds to CDK4 and 6, which prevents them from binding to cyclin D1 (19). This inhibits Rb protein from being phosphorylated and induces cell cycle arrest (19). An initial event in the development of oral carcinomas is a change in the 9p21 locus, which results in the suppression of p16 (19). This suggests that the presence of p16 may be protective. Paradoxically, some OSCCs have demonstrated overexpression of p16, resembling what is typically observed in cervical intraepithelial neoplasia and cervical cancer (20). This may be explained by the fact that human papillomavirus (HPV) inhibits $\mathrm{Rb}$, which leads to a high expression of p16 due to negative feedback regulation (21).

There are conflicting results in studies evaluating p16 expression in oral pre-malignant lesions $(22,23)$, which may be due to the complexity of the mechanism involved. In the present study, the PI of p16 was extremely high in the CLP group, whereas the OD group exhibited the lowest $\mathrm{PI}(\mathrm{P}<0.002)$. This is consistent with the protective characteristics of p16,

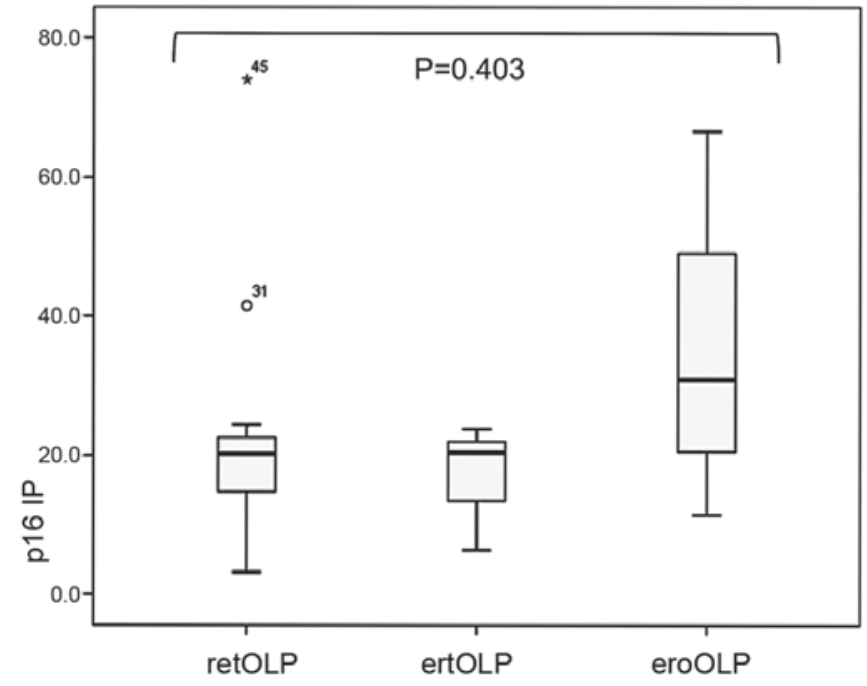

Figure 2. Expression of p16 in retOLP, ertOLP and eroOLP $(\mathrm{P}=0.403)$ retOLP, reticular oral lichen planus; ertOLP, erythematous oral lichen planus; eroOLP, erosive oral lichen planus; PI, positivity index (percentage of positive cells $\mathrm{n} / 500$ ). 


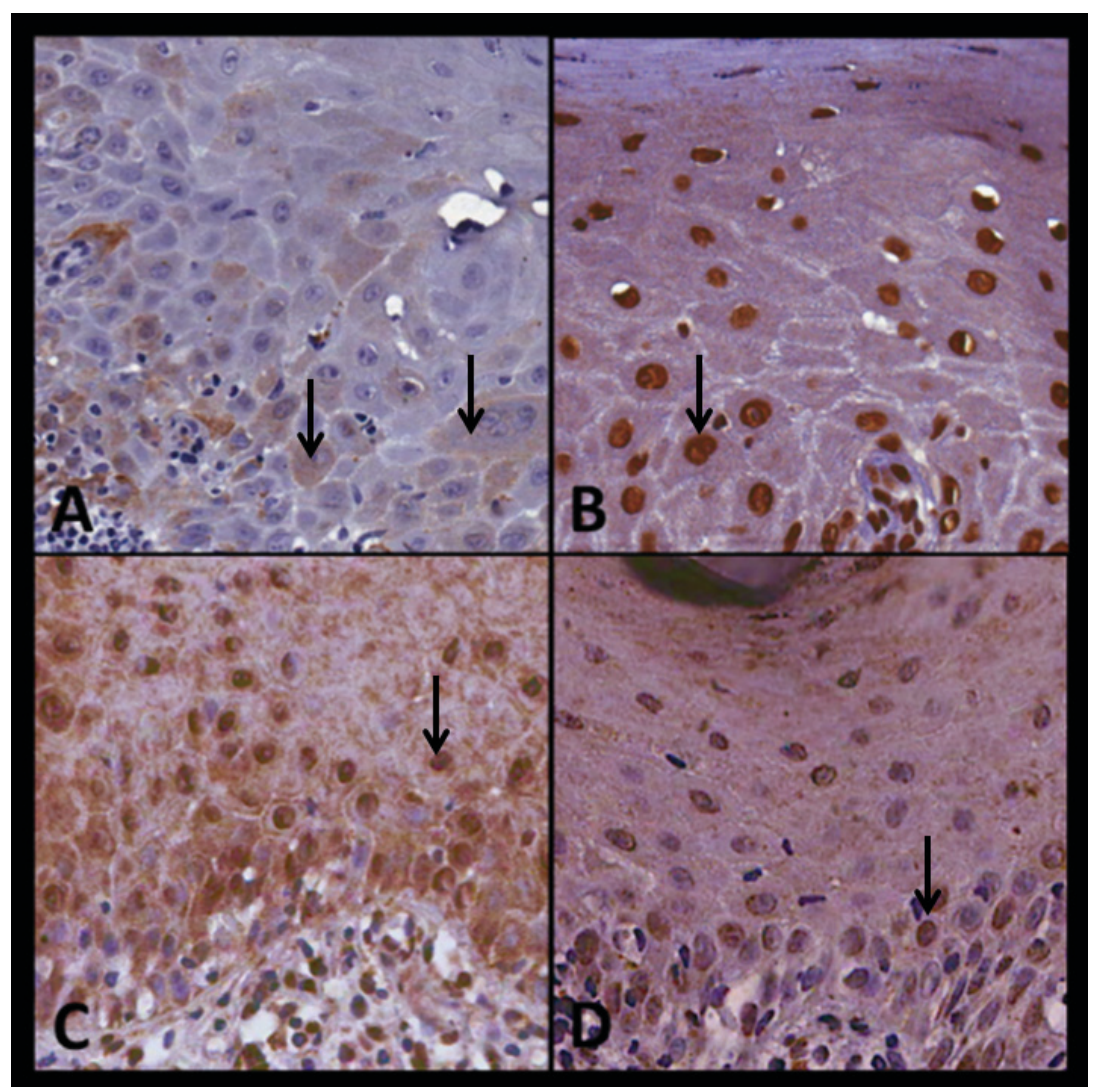

Figure 3. Expression of P16, BUB3, SOX4 and Ki67 in OLP, case no. 22. (A) P16 weak cytoplasmic immunostaining; (B) BUB3 strong nuclear immunostaining; (C) SOX4 moderate nuclear and cytoplasmic immunostaining; (D) Ki67 weak nuclear immunostaining. Arrows indicated positively stained cells. OLP, oral lichen planus; BUB3, budding uninhibited by benzimidazoles 3; SOX4, sex determining region Y-box 4.

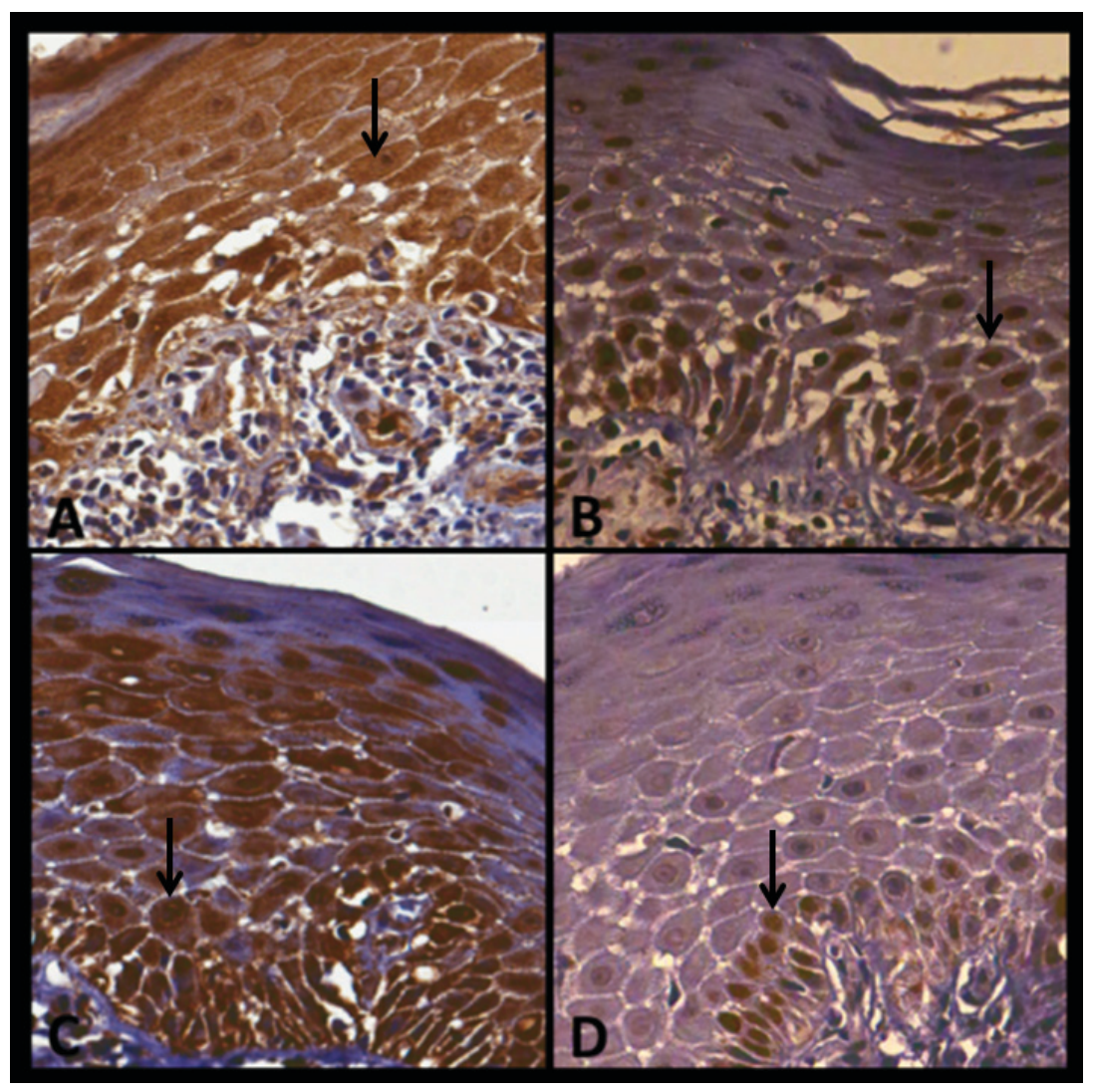

Figure 4. Expression of P16, BUB3, SOX4 and Ki67 in CLP, case no. 12. (A) P16 strong nuclear and cytoplasmic immunostaining; (B) BUB3 strong nuclear immunostaining; (C) SOX4 strong nuclear and cytoplasmic immunostaining; (D) Ki67 weak nuclear immunostaining in basal layer. Arrows indicated positively stained cells. CLP, cutaneous lichen planus; BUB3, budding uninhibited by benzimidazoles 3; SOX4, sex determining region Y-box 4. 


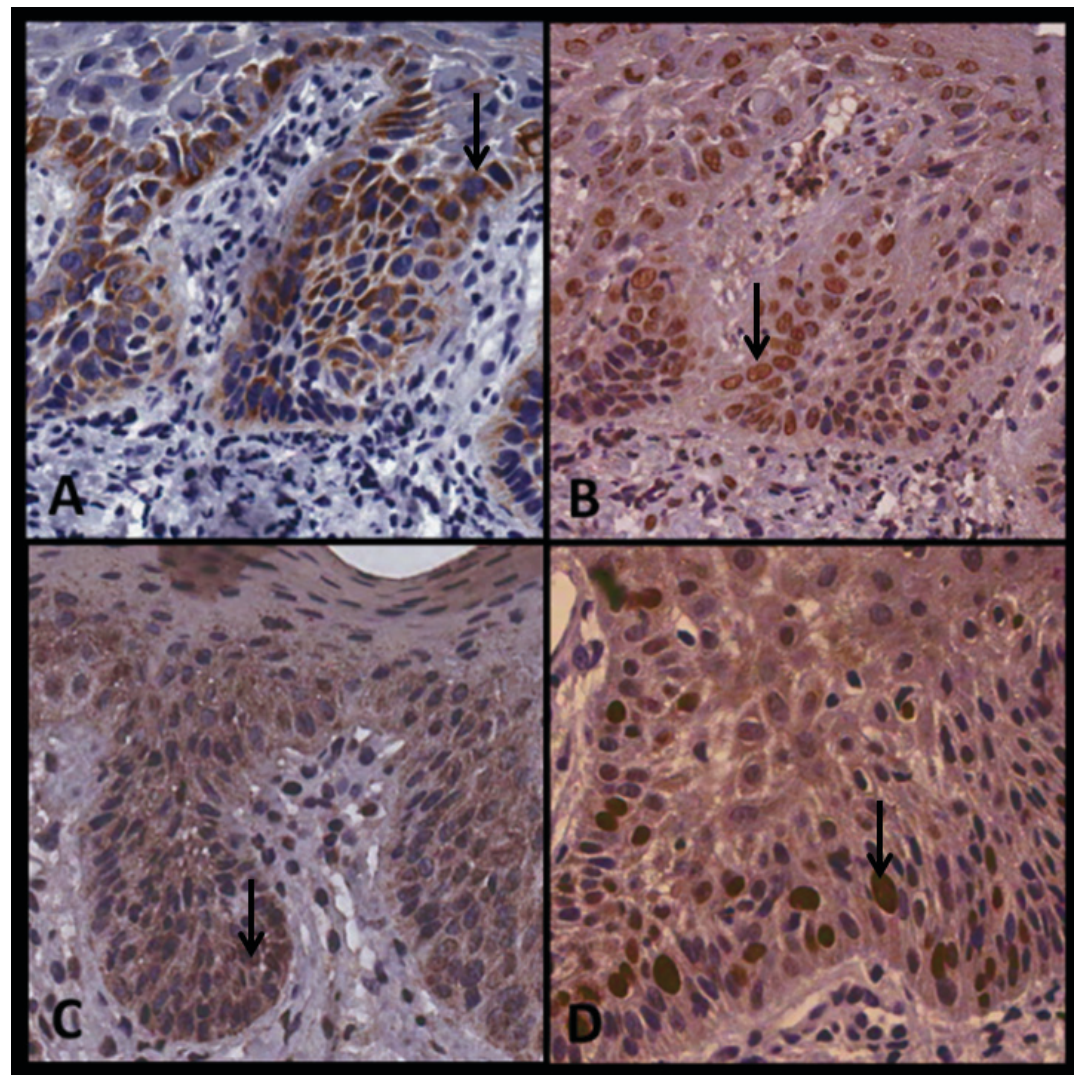

Figure 5. Expression of P16, BUB3, SOX4 and Ki67 in OD, case no. 29. (A) P16 strong nuclear and cytoplasmic immunostaining in basal layer; (B) BUB3 strong nuclear immunostaining; (C) SOX4 moderate nuclear and cytoplasmic immunostaining; (D) Ki67 strong nuclear immunostaining in basal layer. Arrows indicated positively stained cells. OD, oral dysplasia; BUB3, budding uninhibited by benzimidazoles 3; SOX4, sex determining region Y-box 4.

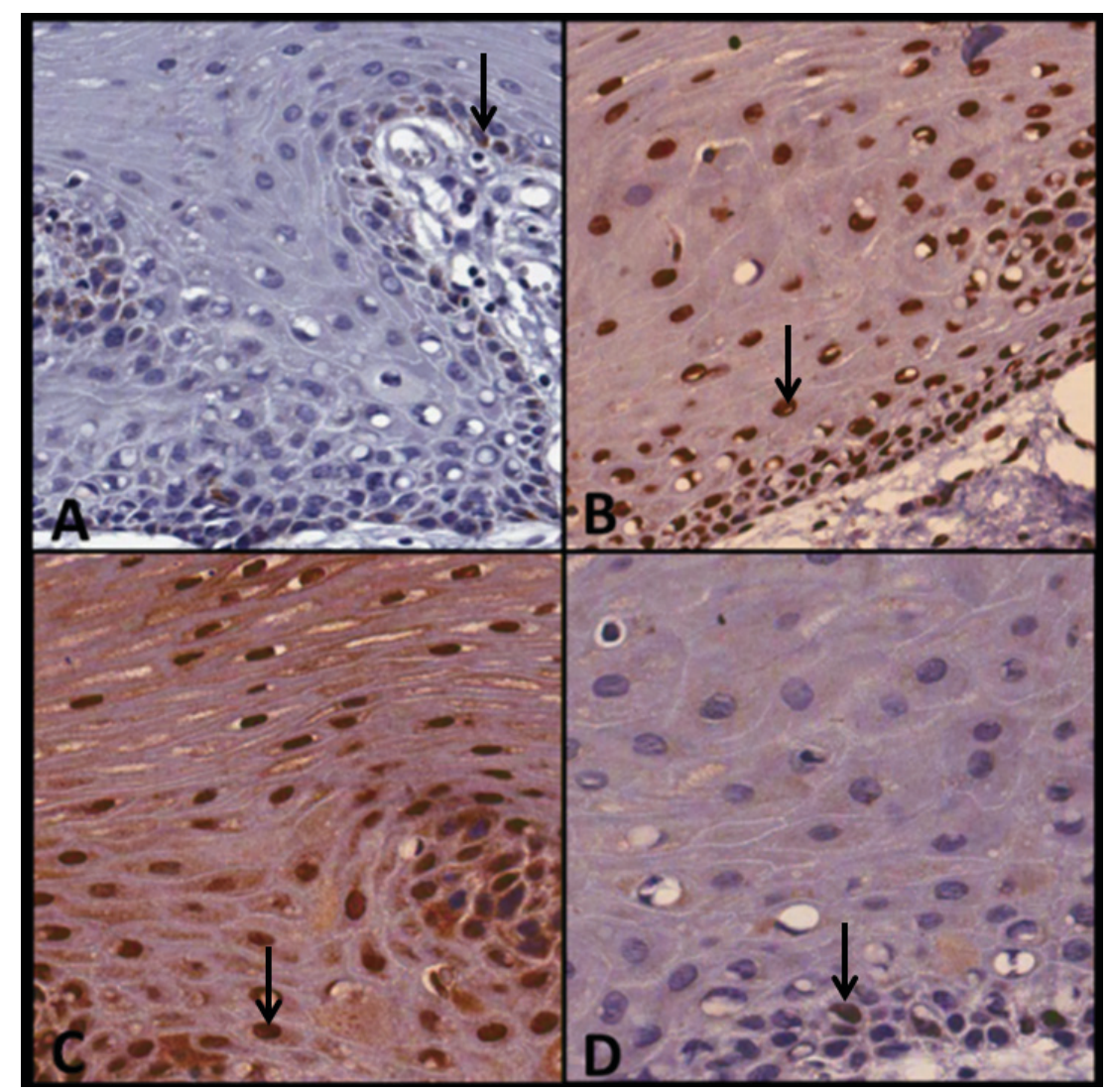

Figure 6. Expression of P16, BUB3, SOX4 and Ki67 in OFH, case no. 23. (A) P16 incipient cytoplasmic immunostaining; (B) BUB3 strong nuclear immunostaining; (C) SOX4 strong nuclear and cytoplasmic immunostaining; (D) Ki67 weak nuclear immunostaining. Arrows indicated positively stained cells. OFH, oral fibrous hyperplasia; BUB3, budding uninhibited by benzimidazoles 3; SOX4, sex determining region Y-box 4. 
considering that CLP reportedly does not have malignant potential (8). To the best of our knowledge, the high expression index of p16 in CLP has not been previously reported. Furthermore, the OLP group revelaed the second-to-highest expression index for $\mathrm{p} 16$. Therefore, the present study classified cytoplasmic staining as p16-positive in order to include all cell mechanisms associated with p16 deregulated expression $(20,21)$.

Oral cancer has multiple etiologies and the major contributing factors include tobacco and alcohol intake (5). The presence of HPV was not evaluated in the present study as previous studies have demonstrated that the majority of OSCC and associated lesions were unrelated to HPV infection (19-21). However, recent findings have indicated a higher prevalence of HPV in associated OSCC lesions indicating a the possibility that some OLP lesions are positive for HPV and also associated with a high p16 expression (22).

However, there is a lack of consistency regarding how to evaluate the expression of p16 in OLP. Distinct criteria impacts the results and the translation of these results in clinical practice for the positive expression of the p16 marker, increasing the variability of the results. For example, in some cases the PI result is dichotomized into positive or negative p16 with different margins in various studies. Of these, certain studies have considered a positive result for cases that have a mark $>70 \%(12,23)$. Only one study demonstrated a high prevalence of HPV in 20 samples of eroOLP using two different molecular techniques. However, the expression of p16 was not assessed (24). In the present study, $20.65 \%$ of cells observed in OLP lesions were positive for p16, suggesting that HPV may be present in OLP.

In the present study, PI can be considered as a robust method as it is standardized, feasible and reproducible for the evaluation of immunoexpression. Future studies aiming to assess the expression p16 in HPV-infected tissues should evaluate such an association. The present results indicated a difference in p16 expression between OLP subtypes, particularly in eroOLP. eroOLP is associated with a higher risk of transformation to OSCC. Notably, a previous study has suggested a correlation between HPV and eroOLP (24). The present findings indicated the difference between OLP subtypes was not statistically significant. However, further investigation into this area is required, taking into account the wide data dispersion compared with other clinical lichen types.

$\mathrm{Ki}-67$ is a nuclear antigen correlated with cellular proliferation and is an extensively explored marker (25). Ki-67 has been evaluated as a predictor of metastasis and as an indicator for the prognosis and recurrence of OSCC $(25,26)$. Furthermore, a positive correlation between Ki-67 expression rate and histological grade has been indicated in studies with non-malignant, pre-malignant and cancerous oral lesions, which suggests its potential as an indirect measure of malignant transformation risk and histologic grading (26). In the present study, Ki-67 expression among the studied lesion groups was the lowest for OFH, followed by CLP, OLP and OD. As expected, OD lesions exhibited the highest PI for this marker. However, OLP lesions exhibited the second-highest PI (11.6\%), which was similar to the expression rate described in previous studies (13 and 13.8\%) $(10,11)$. Notably, eight of the 30 OLP samples presented a Ki-67 expression above the median of that associated with OD $(14,4 \%)$. This may suggest that only specific OLP lesions exhibited malignant potential, which may explain the controversies of current evidence regarding OLP as a premalignant lesion. Furthermore, the expression of Ki-67 was significantly lower between OLP and $\mathrm{OFH}(\mathrm{P}<0.005)$, and between $\mathrm{OD}$ and $\mathrm{OFH}(\mathrm{P}=0.012)$, supporting that $\mathrm{OFH}$ has a reduced risk of transformation, when compared with OD and OLP.

As a member of the mitotic checkpoint complex proteins, BUB3 has an essential role in coupling with other proteins from the same family to prevent cells entering anaphase prematurely (13). The absence of BUB3 has been associated with tumorigenesis in knockout animal models, with widespread aneuploidy and high proliferative potential (27). Similarly, various studies in humans have indicated the association of deregulated expression and mutations in BUB3 with specific types of cancer, including colorectal and lung tumors $(14,15)$. Consistent with this, the results from the present study indicated differential BUB3 expression among the lesions, with the lowest expression in OD and the highest in CLP. These differences were marginally statistically significant, which suggests this marker may be less sensitive in the present assay compared with the two previously discussed markers. This may convey the limited clinical value of this marker on its own. However, further studies are required to investigate the differential expression of BUB3 among different histologic grades of dysplasia.

SOX4 is a member of the SOX transcription factor family (17). Previous findings have suggested that the overexpression of this marker may be associated with cancer development and progression (17). Furthermore, SOX4 has been correlated with the prognosis of breast cancer and recurrence of colorectal cancer (28). However, the role of SOX4 in cancer development has also been debated in the literature. Previous results have identified SOX4 as an oncogene and potential target for cancer vaccinations, suggesting that the loss of SOX4 is associated with loss of tissue viability, whereas its overexpression is associated with immortalized cells (17). However, these conclusions were drawn from in vitro experiments alone. Alternatively, findings have indicated that SOX4 has a role in cell cycle arrest and apoptosis by activating p53, which suggests SOX4 may be an indirect marker of carcinogenesis (28). In this way, upregulated SOX4 expression may be considered an indirect indicator of cell cycle deregulation. The clinical relevance of these hypotheses translates into the usefulness of SOX4 as a biomarker for cancer in soft tissues, particularly in epithelial tissues (29).

In the present study, the expression of SOX4 was high in all groups, particularly in CLP. This finding was unexpected as low expression was anticipated in the CLP group. Additionally, to the best of our knowledge, this is the first time that high SOX4 expression was indicated in CLP. It was hypothesized that the proliferation pattern of epithelium compared with oral mucosa cells varies in lichen planus. In this regard, although the increased expression of SOX4 is typically associated with malignancy $(28,29)$, the present study results suggests that SOX4 overexpression may have a protective role in CLP from malignancies including skin carcinoma. In addition, the durability of SOX4 in CLP may be extremely high, which may lead to an abnormal accumulation within the cell that is 
not associated with malignancy (29). However, further investigation is required to detect the cause for increased SOX4 expression and whether this may clarify the different clinical course of OLP compared with CLP.

In the present study, the lowest level of SOX4 expression was indicated in OLP, followed by OD. Although no statistically significant difference was detected between these groups in the pairwise comparison, the data distribution suggests it is possible that the various clinicopathological characteristics of OLP may be associated with different expressions of SOX4 in each form of OLP. As with p16, this irregular expression may be associated with OLP-specific lesions that may have either a higher or lower likelihood to undergo malignant transformation. Further studies with large and varied populations may aid to elucidate the role of SOX4 in oral carcinogenesis.

The present study did exhibit some limitations. Notably, this was a retrospective study where there was an imbalance in the distribution of the biopsy sites and the clinical lesion subtypes, specifically in the eroOLP group and the grades of dysplasia. This may have affected the results, as there are potential differences between mild and severe dysplasia and between retOLP and eroOLP in the underlying pathophysiology. In addition to the study design, subjects and lesions cannot be followed through time to detect actual malignant transformation of lesions, which would add value in the clinical setting. Furthermore, procedures did not include HPV or double antigen detection, which may provide further useful information. However, double antigen detection is a more technically demanding procedure compared with the single antigen detection, which suggests this may be less feasible in the primary care clinical setting (30). However, for the more equipped facilities, there are promising results on the double-marking of lesions combined with polymerase chain reaction techniques for detection of high-risk oral lesions (30). Furthermore, it was acknowledged that a larger sample size would further support the present findings, particularly considering the variability in the location and type of lesions among some of the studied groups. In addition, the samples used in the present study did not include OSCC biopsies. However, the objective was to evaluate the expression profiles of potentially premalignant lesions as opposed to detecting cancer.

In conclusion, the present study indicated that p16 and Ki-67 expression may suggest that some OLP lesions have an intermediate malignant potential and should be more carefully followed up. The intense SOX4 staining in CLP indicates a different proliferation pattern of epithelium compared with oral mucosa cells and may also be associated with the clinical course in lichen planus. Additional studies are warranted to improve the understanding of the role of BUB3 and SOX4 in OLP.

\section{Acknowledgements}

Not applicable.

\section{Funding}

No funding was received.

\section{Availability of data and materials}

The analyzed data sets generated during the present study are available from the corresponding author on reasonable request.

\section{Authors' contributions}

EAR and AMH made substantial contributions to the conception and design of the study, as well as the acquisition, analysis, and interpretation of all data. They also contributed in drafting the manuscript. DPF made substantial contributions to the design of the study and the analysis of general data, and was responsible for the statistical analysis. LEARF, FFCN and HM made substantial contributions to the design of the study and the analysis of immunohistochemical data. VT and EMK made substantial contributions to the design of the study and the acquisition of oral biopsy data. FPG made substantial contributions to the design of the study and the acquisition of skin biopsy data. RFBA made substantial contributions to the conception and design of the study. DPF, LEARF, FFCN, VT, FPG, EMK, HM and RFBA helped to critically revise the manuscript for important intellectual content. All authors approved the final manuscript and agree to be accountable for all aspects of the work in ensuring that questions related to the accuracy or integrity of any part of the work are appropriately investigated and resolved.

\section{Ethics approval and consent to participate}

All procedures were reviewed and approved by the Institutional Review Board (IRB) from the School of Medicine, University of Brasília (Brasília, Brazil; approval number, CEPFM 042/2010) and conducted in accordance with the principles of the Declaration of Helsinki. Informed consent was obtained in the majority of cases; however, some patients were not available following several contact attempts or had experienced mortality. Therefore, the IRB approved not having the formal consent in these cases.

\section{Consent for publication}

Not applicable.

\section{Competing interests}

The authors declare that they have no competing interests.

\section{References}

1. Ferlay J, Shin HR, Bray F, Forman D, Mathers C and Parkin DM: Estimates of worldwide burden of cancer in 2008: GLOBOCAN 2008. Int J Cancer 127: 2893-2917, 2010.

2. Dias GS and Almeida AP: A histological and clinical study on oral cancer: Descriptive analyses of 365 cases. Med Oral Patol Oral Cir Bucal 12: E474-E478, 2007.

3. Amagasa T, Yamashiro M and Uzawa N: Oral premalignant lesions: From a clinical perspective. Int J Clin Oncol 16: 5-14, 2011.

4. Omal P, Jacob V, Prathap A and Thomas NG: Prevalence of oral, skin, and oral and skin lesions of lichen planus in patients visiting a dental school in southern India. Indian J Dermatol 57: 107-109, 2012.

5. Barnes L, Eveson JW, Reichart P and Sidransky D: Pathology and genetics of head and neck tumours. WHO Classif Tumour: $163-75,2005$ 
6. van der Meij EH, Mast $\mathrm{H}$ and van der Waal I: The possible premalignant character of oral lichen planus and oral lichenoid lesions: A prospective five-year follow-up study of 192 patients. Oral Oncol 43: 742-748, 2007.

7. Xue JL, Fan MW, Wang SZ, Chen XM, Li Y and Wang L: A clinical study of 674 patients with oral lichen planus in China. J Oral Pathol Med 34: 467-472, 2005.

8. Eisen D: The clinical features, malignant potential, and systemic associations of oral lichen planus: A study of 723 patients. J Am Acad Dermatol 46: 207-214, 2002.

9. Rosa EA, Lia EN, Macedo SB and Amorim RF: In situ carcinoma developed over oral lichen planus: A case report with analysis of BUB3, p16, p53, Ki67 and SOX4 expression. J Appl Oral Sci 23 442-447, 2015.

10. Acay RR, Felizzola CR, de Araújo N and de Sousa SOM: Evaluation of proliferative potential in oral lichen planus and oral lichenoid lesions using immunohistochemical expression of p53 and Ki67. Oral Oncol 42: 475-480, 2006

11. Zargaran M, Jamshidi S, Eshghyar N and Moghimbeigi A: Suitability/unsuitability of cell proliferation as an indicator of malignant potential in oral lichen planus: An immunohistochemical study. Asian Pac J Cancer Prev 14: 6979-6983, 2013.

12. Nankivell P, Williams H, Webster K, Pearson D, High A, MacLennan K, Senguven B, McConkey C, Rabbitts P and Mehanna $\mathrm{H}$ : Investigation of p16(INK4a) as a prognostic biomarker in oral epithelial dysplasia. J Oral Pathol Med 43: 245-249, 2014.

13. Han JS, Vitre B, Fachinetti D and Cleveland DW: Bimodal activation of BubR1 by Bub3 sustains mitotic checkpoint signaling. Proc Natl Acad Sci USA 111: E4185-E4193, 2014.

14. Mukherjee A, Joseph C, Craze M, Chrysanthou E and Ellis IO: The role of BUB and CDC proteins in low-grade breast cancers. Lancet 385 (Suppl 1): S72, 2015.

15. de Voer RM, Geurts van Kessel A, Weren RD, Ligtenberg MJ, Smeets D, Fu L, Vreede L, Kamping EJ, Verwiel ET, Hahn MM, et al: Germline mutations in the spindle assembly checkpoint genes BUB1 and BUB3 are risk factors for colorectal cancer. Gastroenterology 145: 544-547, 2013.

16. Bangur CS, Switzer A, Fan L, Marton MJ, Meyer MR and Wang T: Identification of genes over-expressed in small cell lung carcinoma using suppression subtractive hybridization and cDNA microarray expression analysis. Oncogene 21: 3814-3825, 2002.

17. Friedman RS, Bangur CS, Zasloff EJ, Fan L, Wang T, Watanabe Y and Kalos M: Molecular and immunological evaluation of the transcription factor SOX-4 as a lung tumor vaccine antigen. J Immunol 172: 3319-3327, 2004.

18. van der Meij EH and van der Waal I: Lack of clinicopathologic correlation in the diagnosis of oral lichen planus based on the presently available diagnostic criteria and suggestions for modifications. J Oral Pathol Med 32: 507-512, 2003.

19. Ai L, Stephenson KK, Ling W, Zuo C, Mukunyadzi P, Suen JY, Hanna E and Fan CY: The p16 (CDKN2a/INK4a) tumor-suppressor gene in head and neck squamous cell carcinoma: A promoter methylation and protein expression study in 100 cases. Mod Pathol 16: 944-950, 2003.

20. Abrahao AC, Bonelli BV, Nunes FD, Dias EP and Cabral MG: Immunohistochemical expression of p53, p16 and hTERT in oral squamous cell carcinoma and potentially malignant disorders. Braz Oral Res 25: 34-41, 2011
21. Murphy N, Ring M, Heffron CC, King B, Killalea AG, Hughes C, Martin CM, McGuinness E, Sheils O and O'Leary JJ: p16INK4A, CDC6, and MCM5: Predictive biomarkers in cervical preinvasive neoplasia and cervical cancer. J Clin Pathol 58: 525-534, 2005.

22. Broglie MA, Jochum W, Förbs D, Schönegg R and Stoeckli SJ: Brush cytology for the detection of high-risk HPV infection in oropharyngeal squamous cell carcinoma. Cancer Cytopathol 123: 732-738, 2015.

23. Salehinejad J, Sharifi N, Amirchaghmaghi M, Ghazi N, Shakeri MT and Ghazi A: Immunohistochemical expression of p16 protein in oral squamous cell carcinoma and lichen planus. Ann Diagn Pathol 18: 210-213, 2014.

24. Jontell M, Watts S, Wallström M, Levin L and Sloberg K: Human papilloma virus in erosive oral lichen planus. J Oral Pathol Med Off Publ Int Assoc Oral Pathol Am Acad Oral Pathol 19: 273-277, 1990

25. Perisanidis C, Perisanidis B, Wrba F, Brandstetter A, El Gazzar S, Papadogeorgakis N, Seemann R, Ewers R, Kyzas PA and Filipits M: Evaluation of immunohistochemical expression of p53, p21, p27, cyclin D1, and Ki67 in oral and oropharyngeal squamous cell carcinoma. J Oral Pathol Med 41: 40-46, 2012

26. Birajdar SS, Radhika M, Paremala K, Sudhakara M, Soumya M and Gadivan M: Expression of Ki-67 in normal oral epithelium, leukoplakic oral epithelium and oral squamous cell carcinoma. J Oral Maxillofac Pathol 18: 169-176, 2014.

27. Morais da Silva S, Moutinho-Santos T and Sunkel CE: A tumor suppressor role of the Bub3 spindle checkpoint protein after apoptosis inhibition. J Cell Biol 201: 385-393, 2013.

28. Pan X, Zhao J, Zhang WN, Li HY, Mu R, Zhou T, Zhang HY, Gong WL, Yu M, Man JH, et al: Induction of SOX4 by DNA damage is critical for p53 stabilization and function. Proc Natl Acad Sci USA 106: 3788-3793, 2009.

29. Watanabe M, Ohnishi Y, Wato M, Tanaka A and Kakudo K: SOX4 expression is closely associated with differentiation and lymph node metastasis in oral squamous cell carcinoma. Med Mol Morphol 47: 150-155, 2014.

30. Linxweiler M, Bochen F, Wemmert S, Lerner C, Hasenfus A, Bohle RM, Al-Kadah B, Takacs ZF, Smola S and Schick B: Combination of p16INK4a/Ki67 immunocytology and hpv polymerase chain reaction for the noninvasive analysis of HPV involvement in head and neck cancer. Cancer Cytopathol 123: 219-229, 2015.

This work is licensed under a Creative Commons Attribution-NonCommercial-NoDerivatives 4.0 International (CC BY-NC-ND 4.0) License. 